\title{
MedienPädagogik
}

www. medienpaed.com

\section{Didaktische Konzeption von Serious Games: Zur Verknüpfung von Spiel- und Lernangeboten}

Michael Kerres, Mark Bormann und Marcel Vervenne

\begin{abstract}
Serious Games versuchen Spielen mit Lernen zu verbinden. Der Beitrag problematisiert die Machbarkeit einer solchen Verknüpfung in digitalen Welten und diskutiert verschiedene didaktische Konzepte einer Nutzbarmachung digitaler Spiele für das Lernen und Lehren. Ausgehend von einer Analyse des Erlernens von Spielen werden drei didaktische Konzepte von Serious Games im Hinblick auf ihre didaktischen Potenziale untersucht: (1) der unmittelbare Transfer von Wissen, das im Spiel erworben wird (keine zusätzliche Didaktisierung), (2) Didaktisierung durch Einbettung des Spiels in einer Lernsituation oder (3) Didaktisierung durch Einbettung von Lernaufgaben in ein Spiel. Ergebnisse zur Nutzung von Spielen, bei denen Lernaufgaben in eine Spielwelt eingebettet sind, zeigen, dass Personen die Aufnahme von Wissen in einem expliziten Lernmodus eher zu reduzieren versuchen, um möglichst zügig wieder in den Spielmodus zu wechseln. Diskutiert werden die Konsequenzen dieser Problematik für die didaktische Konzeption von Serious Games.
\end{abstract}

\section{$1 \quad$ Einleitung}

Spielen ist ganz essentiell mit Lernprozessen verbunden. Zugleich entzieht sich das Spiel einer einfachen Instrumentalisierung für didaktische Zwecke: Spielen ist nach Huizinga eine freiwillige Aktivität, die um ihrer selbst willen ausgeführt wird (Fromme 2007; Huizinga 2004; Oerter 1999; Schrammel und Mitgutsch 2009).

Betrachtet man diese Paradoxie, stellt sich die Frage der Machbarkeit einer Nutzbarmachung von digitalen Spielen für didaktische Szenarien. Die grundsätzliche Frage lautet dabei, inwiefern in der Spielwelt implizites und explizites Lernen stattfindet und dies mit einem Kompetenzerwerb einhergeht, der sich auf Wissensdomänen und Anwendungssituationen ausserhalb der Spielwelt überträgt.

Spielen erfordert immer, sich eine Spielidee anzueignen, Informationen über Spielelemente, -ablauf und -ziel aufzunehmen und die Anwendung eines zum Teil relativ komplexen Regelwerkes zu erlernen. Spielen kann damit als ein anspruchsvoller Lernprozess betrachtet werden. Es dauert zumeist mehrere Durchgänge bis ein Spiel beherrscht wird. Manche Spiele sind über Jahre und Jahrzehnte zu spielen, bevor sich «Meisterschaft» einstellt. Zugleich - und das macht (auch) das Faszinierende des Spiels aus - wird das Spiel gerade nicht als «Lernsituation» erlebt. 
Im Folgenden soll das Verhältnis von Spielen und Lernen analysiert werden, um die Potenziale des Game-Based Learning für Kompetenzerwerb abzuschätzen und Modelle der didaktischen Konzeption von Serious Games formulieren zu können. Ausgehend von der Frage, wie überhaupt in Spielen gelernt wird, wird untersucht, welche Art von Kompetenzerwerb mit Spielen möglich ist.

\section{Das Erlernen von Spielen}

Das Erlernen von Spielen bedeutet einen zum Teil langwierigen Lernprozess, der entlang der Dimension des Anfängers, über Fortgeschrittenen zum Expert/in beschrieben werden kann und je nach Komplexität des Regelwerkes zum Teil viele Spieldurchgänge erfordert. Nach Behr, Klimmt \& Vorderer (2008) ist der Unterhaltungswert eng mit leistungsorientiertem Verhalten, dem Wettbewerb um einen Standard, verbunden. Es zeigt sich, dass mittelschwere, bewältigbare Spielsituationen als unterhaltsam erlebt werden.

Garris et al. (2002) beschreiben das Erlernen von Spielen als einen Spielzyklus aus Verhalten des Spielers, Rückmeldung und Bewertung des Spiels mit erneuter Zielsetzung. Der Kompetenzerwerb geschieht in einem mehrfachen Durchlauf dieses Spielzyklus. Die Person führt einen Spielzug aus und erhält eine Reaktion. Sie bewertet die Situation und entscheidet sich für einen weiteren Spielzug. Das Set der Regeln, nach denen das Spiel funktioniert, ist dabei keineswegs von Beginn an vollständig bekannt. Die Person erlernt im Umgang mit dem Spiel, was wo wie zu tun ist bzw. getan werden kann. Dabei entwickelt sie ein zunehmend komplexeres Bild von der Spielwelt und baut eine zunehmend verästelte, mentale Karte der Anwendung auf.

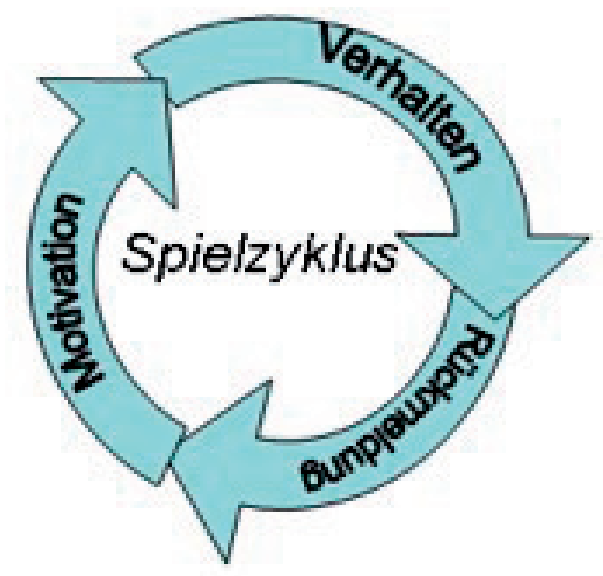

Abb. 1: Der Spielzyklus (nach Garris et al., 2002) 
Gerade in digitalen Spielwelten mit hoher Spieldynamik erinnert der Spielzyklus damit an das Prinzip der operanten Konditionierung sensu Skinner: Die Person «emittiert» ein Verhalten; ähnlich wie die Taube in der Skinnerbox versucht sie herauszufinden, wie sie eine Futterpille erhält. Das Verhalten der Person wird durch die Rückmeldung des Systems unmittelbar gesteuert. Gerade bei digitalen Spielwelten, die auf Schnelligkeit angelegt sind, mit Spielzyklen von nur wenigen Sekunden, ist ein Vorgehen nach "Versuch-und-Irrtum», also ohne intensivere Planung und Auswertung von Spielzügen, eine typische und über lange Strecken durchaus adäquate Spielstrategie. Zugleich findet an bestimmten Stellen des Spielverlaufs auch eine kognitive Auseinandersetzung mit Spielstand, die Reflexion des Spielverlaufs und die Planung des weiteren Vorgehens statt (wie Bopp (2008) sie beschrieben hat). Diese Planungs- und Entscheidungssituationen werden beim Spiel eher als Einschnitt im Spielfluss erlebt, als ein Zustand, den man eher verlassen möchte.

Zum Erlernen eines Spiels muss die Person also zunächst die Spielidee verstehen, sie muss die Bestandteile und Regeln des Spiels kennen und benennen können (deklaratives Wissen). Im Spielzyklus wendet sie dieses Wissen dann an und optimiert es mit jedem Spielzyklus. Ein wichtiges Element, das zugleich auch Spass vermittelt, ist dabei das Ausprobieren: Ich muss zuvor nicht alles wissen, ich kann bestimmte Spielzüge ausprobieren und erhalte sofort Rückmeldungen. In digitalen Spielwelten mit hoher Komplexität sind die Regeln dabei nicht (vollständig) bekannt. Die Person weitet in vielen Runden ihr Wissen immer mehr aus. Umso mehr Wissen die Person erwirbt, umso erfolgreicher kann sie sich in der Welt bewegen und steht vor zunehmend schwerer werdenden Herausforderungen, die ihre Kompetenz unter Beweis stellt. Dieses «erhebende» Gefühl des Nachweises zunehmender Kompetenz - im Grunde ein Nebenprodukt des Spielens - ist eine wesentliche Motivationsquelle, wie Behr, Klimmt \& Vorderer (2008) aufzeigen.

Dabei zeigt sich, dass reines «Versuch-und-Irrtum» für den Kompetenzaufbau bei digitalen Spielen in der Regel nicht hinreichend ist und Spielende schnell demotivieren würde. Aus diesem Grund sind in den Spielwelten unterschiedliche Lernhilfen eingebaut:

- Tutorials erläutern die Grundlagen der Spielmechanik. Sie sind im Grunde aufgebaut, wie ein «Lehrbuch», und können damit als didaktisierte Lernhilfe bezeichnet werden.

- In Foren findet man Hinweise für die Bewältigung bestimmter Spielsituationen und können andere Spieler um Hilfe gefragt werden. Hier sind zum Teil auch Tricks und "Cheats» zu finden, wie bestimmte Situationen bewältigt werden können.

- Einführungsmissionen zeigen in einer vereinfachten und reduzierten Spielumgebung, wie das Spiel funktioniert. Dabei taucht in der Regel ein NPC (non- 
player character) als Avatar auf, der die Person begleitet auf ihren ersten Wegen und Elemente der Umgebungen erläutert.

Eine Grundregel des Game Design lautet, dass ein Spiel möglichst schnell spielbar sein muss. Je länger die erforderliche Einweisung, je höher ist die Gefahr des Abspringens - für kommerzielle Anbieter offensichtlich ein essentielles Kriterium im Spieldesign (vgl. Salen und Zimmerman 2003). Es zeigt sich, dass Spielende längere «Belehrungen» deutlich meiden.

In solchen komplexen Welten geht es also nicht mehr nur darum, zuvor benannte Regeln anzuwenden, also deklaratives Wissen in prozedurales Wissen zu überführen. Es geht vielmehr darum, in der Interaktion mit dem System prozedurales Wissen zu generieren. Die Logik, die der Programmierung des Spiels zugrunde lag, ist für den Spieler verborgen; sie ist «im» Spielen zu rekonstruieren. Vielfach ist dieses Wissen, das im Spielen implizit erworben wird, schwerlich explizierbar. Die Person weiss, wie sie bestimmte Situationen zu meistern hat, kann dies aber kaum verbalisieren.

Das Wissen, das die Person in solchen Spielwelten erwirbt, kann als eine Sammlung von "Skripts» (Schank und Abelson 1977) betrachtet werden. In einem Skript ist das Wissen abgelegt, wie ein bestimmter Typ von Situation zu bewältigen ist, was dort zu beachten ist und welche Reihenfolge von Handlungen typischerweise angemessen ist. Dieses Wissen wird durch mehrfache Anwendung routinisiert und läuft automatisch ab, sobald eine Situation einem bestimmten Skript zugeordnet werden kann. Expertise zeichnet sich dadurch aus, dass die Person über eine ganze Reihe ausgearbeiteter Skripte verfügt, und diese hochgradig routinisiert in ihrem Verhaltensrepertoire verfügbar hat. Bei komplexen Spielwelten muss die Person also zum einen Regeln anwenden, zum anderen muss sie in bestimmten Situationen, in denen bisheriges Wissen versagt, neues Wissen generieren.

Behr, Klimmt \& Vorderer (2008) weisen auf, dass Spielspass entsteht, wenn die Person sich in Spielwelten mittlerer Schwierigkeit bewegt. Wird das Spiel als zu leicht oder zu schwer erlebt, bestärkt das die Tendenz ein Spiel abzubrechen. Deswegen muss das Level Design sich jeweils an den Stand der Spielenden anpassen. Auch hier eine Parallele zu Lern- und Leistungsmotivation: Denn bei der Bearbeitung von (Lern-) Aufgaben stellt sich ein Gefühl von Stolz über die erbrachte Leistung bekanntlich ebenfalls bei mittelschweren Aufgaben ein. Das Prinzip der "Passung» beschreibt, wie im Unterricht Aufgaben steigender Schwierigkeit an das Leistungsniveau des Lernenden angepasst werden sollten (Heckhausen 1989).

Klimmt (2005) unterscheidet zwischen problemlösendem und explorativem Handeln im Spiel. Exploratives Handeln kommt (erst) zum Einsatz, wenn zusätzliche Informationen oder Objekte benötigt werden, um bestimmte Probleme (Missionen etc.) bewältigen zu können. Der Zustand der Problemlösung beinhaltet dagegen das Eintauchen in die Spielwelt. Wir könnten sagen, dass hier implizites Lernen im 
Spielzyklus stattfindet. Nur wenn die Person in der Spielwelt nicht weiterkommt, wechselt sie zum explorativen Handeln und damit zu einem Skript des expliziten Lernens, und zwar nur solange wie notwendig, um wieder in die Spielwelt einzutauchen und dort das neue Wissen zur «Problemlösung» anwenden zu können. Eine Spielwelt, die lange Phasen des expliziten Lernens erfordert, wäre demnach für Spielende wenig attraktiv (s.a. Wolling, Quandt, und Wimmer 2008).

Um die Notwendigkeit eines Umschaltens in den Modus expliziten Lernens zu verhindern, schlägt Bopp (2005) ein didaktisch-immersives Spieldesign vor. Es zeichnet sich dadurch aus, dass das Spiel eine Lernhilfe anbietet ohne das der Spieler subjektiv in den Lernmodus umschalten muss: Er bleibt «im» Spiel. Bopp nennt folgende Möglichkeiten, wie in der Gestaltung der Spielumgebung Lernunterstützung gegeben werden kann:

a. Objekte können so gestaltet werden, dass sie einen Aufforderungscharakter für bestimmte Handlungen hervorrufen. So wird ein Objekt, das sich bewegt oder verändert, relativ wahrscheinlich angeklickt. Wenn das System also feststellt, dass die Person an einer bestimmten Stelle nicht weiter kommt, kann das Objekt etwa durch Bewegung oder Farbveränderung «auf sich aufmerksam machen». Ungünstig wäre dagegen eine Belehrung über ein Popup-Fenster, das die Person auf eine bestimmte Handlung hinweist.

b. Das Level-Design legt bestimmte Stufen steigender Schwierigkeit im System fest, die jeweils durchlaufen werden müssen bevor die nächst schwierigere Stufe erreicht wird. Hierbei kommt etwa das motivationspsychologische «Prinzip der Passung» (Heckhausen), das «Mastery-Learning» (Bloom) oder das «Scaffolding» (Vygotsky) zum Tragen. Die Person wird behutsam in immer schwierigere Situationen geleitet und weitet dabei ihre Kompetenzen immer mehr aus.

c. In Spielwelten kann die Person anderen Spieler/innen oder auch «non-player character» (NPC) begegnen: Avatare, die wie Mitspieler agieren, aber vom System gesteuert werden. Die Person kann etwa beobachten, wie andere Personen oder NPCs bestimmte Situationen meistern, oder ein NPC kann dem Spieler gezielt Hinweise geben, ohne dass diese vom Spieler in der Regel als pädagogisch konnotiert erlebt werden: «Schau doch mal hinter das Haus ...».

Damit wird deutlich, dass eine wesentliche Aufgabe des Spieldesign darin besteht, Lernprozesse beim Spielen anzuregen. Zugleich besteht die Herausforderung darin, das Spiel so zu gestalten, dass der Lernprozess «im» Spiel stattfinden kann. Die Person kann viele Dinge durch Versuch-und-Irrtum erfahren, es müssen aber zugleich durch gestalterische Elemente bestimmte Lernprozesse ermöglicht werden, die darüber hinaus gehen. Eher ungern und für begrenzte Dauer wechselt die Person in den Modus expliziten Lernens, in dem das eigene Handeln zum Gegenstand der Reflexion wird Informationen aktiv aufgesucht werden. 


\section{Lernen «mit» Spielen}

Ein Spiel zu erlernen ist ein iterativer Vorgang, der je nach Komplexität viele Durchgänge erfordern kann, bis ein gewisser Grad an Expertise vorliegt. Werden aber nun im Zuge dieses Kompetenzerwerbs auch Kenntnisse und Fertigkeiten erworben, die ausserhalb des Spiels angewendet werden können und damit Lerntransfer ermöglichen? Diese Erwartung, die die Protagonisten des GBL, wie Prensky (2003) und Gee (2003), mit Game-Based Learning letztlich verknüpfen, soll im Folgenden anhand von unterschiedlichen Konzepten der Nutzung von Spielen für Lernzwecke diskutiert werden.

\subsection{Lernen mit Simulationen}

In einer Simulation kann der Umgang mit dem System auf "spielerische Weise» erlernt werden: Lernende können Dinge gefahrlos ausprobieren, die Effekte bestimmter Handlungen testen und unmittelbar Rückmeldung erhalten. Die Forderung nach Lerntransfer ist in Simulationen auf den ersten Blick leicht eingelöst: Die Simulation beinhaltet eine Nachbildung eines Systems. Beherrscht die Person die Simulation sollte sie auch in der Lage sein, das reale System zu meistern.

Bei Simulationen wird vor allem prozedurales, aber auch deklaratives Wissen aufgebaut. Die Simulation versucht für das Lernen wesentliche Aspekte der Realität ab- bzw. nachzubilden. Auf diese Weise, so die Hoffnung, soll die Transferierbarkeit auf die «Echtsituation» ermöglicht werden. Dies entspricht der traditionellen Forderung der Theorie der identischen Elemente von Thorndike, wonach Lerntransfer dann gelingt, wenn Lern- und Anwendungssituation hinreichend ähnlich sind (Mähler und Stern 2006).

Dieser Transfereffekt sollte demnach zumindest bei Simulationen möglich sein, bei denen es um ein technisches System geht. Die Simulation kann dem Realsystem relativ weit folgen, weil es sich um ein als technisch konstruiertes Artefakt handelt, dessen Funktions- und Konstruktionsprinzipien grundsätzlich bekannt sind. So spielen in der Ausbildung von Flugzeugpiloten Simulatoren eine grosse Rolle. Freilich bilden echte Flugsimulatoren der Pilotenausbildung das Verhalten von Flugzeugen sehr viel weitreichender ab als ihre «spielerischen» Pendants auf PCs. Dieses Szenario würde damit der Forderung von Thorndike entsprechen, wonach Lern- und Anwendungssituation eine hohe Ähnlichkeit aufweisen sollten. Dabei bleibt offen, was die «Ähnlichkeit» von Situationen letztlich ausmachen muss, um einen Transfereffekt sicherzustellen. Es sind sicherlich nicht die Oberflächenmerkmale der Situation; die grundlegenden, lernrelevanten Merkmale der Situation zu bestimmen, ist nicht trivial. Auch zu bedenken ist, dass ein negativer Lerntransfer eintreten kann, d.h. eine gelernte Routine in der Spiel- oder Lernwelt behindert die Anwendung in der Realsituation, wenn diese sich in bestimmter Hinsicht, ggfs. auch nur gering, unterscheidet. 
Bei einem nicht-technischen System ist die Übereinstimmung von Lern- und Anwendungssituation wesentlich geringer. Bei Unternehmensplanspielen kann die Simulation das reale System nur nachahmen, da dessen Konstruktionsprinzipien unbekannt sind. Regeln für z. B. die erfolgreiche Unternehmensführung können zwar auf der Grundlage von Modellen plausibel formuliert werden, aber kaum in Funktionsgleichungen überführt werden, die zuverlässig z. B. reale Unternehmensentwicklungen abbilden. So ist auch keineswegs sichergestellt, dass Personen, die gelernt haben, eine solche Simulation erfolgreich zu bewältigen, auch in echten Unternehmen erfolgreich sein werden: Soziale Realität ist «überdeterminiert».

Gleichwohl ist der Lerneffekt etwa von Unternehmensplanspielen mehrfach nachgewiesen und durchaus plausibel (Blötz 2002; vgl. Geilhardt und Mühlbradt 1995; Mandl u. a. 2001) Dieser hängt - neben der Qualität der Simulation - vor allem von der Lernorganisation und der Einbettung des Spiels in eine Lernsituation ab: So werden diese z. B. über längere Zeiträume hinweg in Lerngruppen auch als Fernplanspiele organisiert. Dabei findet in der Regel ein Austausch zwischen den Lernenden statt und die Personen können viel über grundlegende Mechanismen und Funktionsprinzipien von Märkten und Unternehmen erfahren. Planspiele können somit über Reflexion und Kommunikation wichtige Lernprozesse und Wissensaustausch anregen, auch ohne dass die Simulation die Funktion eines Unternehmens «wirklich» zuverlässig abbildet.

Eine Frage wäre, inwiefern Simulationen überhaupt «Spiele» sind. Denn mit dem Anspruch, Realität nachzubilden bzw. nachzuahmen, fehlt ihnen das Kriterium, wonach ein Spiel «anders» ist als alltägliche Routine-Situationen. Für den Piloten ist der Flugsimulator sicherlich eine Trainingsanwendung (und kein Spiel!). Für den ambitionierten Spieler dagegen eine aussergewöhnliche, den Alltag unterbrechende, spielerische Erfahrung.

\subsection{Lernen mit digitalen Spielwelten}

Im Vordergrund der Diskussion über Game-Based Learning stehen digitale Spielwelten, die auf Phantasiewelten, die abenteuerliche oder bedrohliche Phänomene beinhalten. Diese Fremdartigkeit löst Neugier aus und die Motivation, sich in dieser Welt zurechtzufinden. Das deklarative Wissen, das in diesen Spielwelten erworben wird, ist in der Regel deutlich auf diese Spielwelt bezogen, und insofern für einen Transfer eher wenig bedeutsam. Prozedurales Wissen würde sich auf Fertigkeiten etwa im Umgang mit Objekten in der Spielwelt beziehen, und es stellt sich die Frage, ob und wie sich diese Prozeduren in Anwendungskontexten nutzen lassen.

Die Protagonisten des GBL verweisen vor allem auf den Erwerb von generischen Strategien und metakognitiven Fertigkeiten, wie z. B. den Umgang mit komplexen Situationen, die Informationssuche unter Zeitdruck, die Koordination mit anderen Mitspielern und schliesslich die Reaktionsgeschwindigkeit sowie die Hand-Auge- 
Koordination. Ebenso wird auf die Entwicklung bestimmter - positiver - Einstellungen verwiesen, wie z.B. der Umgang mit Unsicherheit, ein höheres Selbstbewusstsein und positive Selbstwirksamkeitserwartungen.

Klimmt (2005) nimmt etwa an, dass im Umgang mit einer virtuellen Welt Selbstwirksamkeit erworben wird: Die Person erlebt, dass sie - durch ihr zunehmendes Regelwissen - eine zunächst kaum durchschaubare, komplexe Welt immer mehr kontrollieren kann und dabei ggfs. auch soziale Anerkennung in der virtuellen oder realen Umwelt erfährt. John Hagel \& John Seely Brown (2009) empfehlen «World of Warcraft» sogar als Trainings- wie auch Personalselektionsinstrument für künftiges Führungspersonal:

Companies seeking to thrive in a world of increasing uncertainty and accelerating change will need to foster this disposition among their own executive team and employees. They would be well advised to take a closer look at World of Warcraft, both in terms of the approach taken to foster this disposition and as a potential recruiting ground for employees who can bring this attitude and approach into the company.

Es scheint durchaus denkbar, dass diese Kompetenzen in Spielen erworben werden können. Es liegen allerdings keine hinreichenden empirischen Belege für diese weit reichenden Behauptungen vor und auf Grundlage der theoretischen Diskussion über Lerntransfer erscheint Skepsis zunächst weiter angemessen (vgl. Mähler und Stern 2006). Gerade in den 1990er-Jahren ist das Problem des «trägen Wissens» als zentrales Problem von Unterricht und Lernen aufgegriffen worden: «Spontaner» Lerntransfer tritt eher selten ein als vielfach vermutet wird. Lerntransfer entsteht systematisch nur, wenn bereits die Lernsituation durch ein bestimmtes didaktisch-methodisches Arrangement auf die Anwendung hin gestaltet ist: Die Ansätze des Lernens mit Fällen, Problemen oder Projekten tragen etwa dazu bei, einen solchen Lerntransfer zu fördern (s. etwa Mandl und Kopp 2006).

Es bleibt damit die Frage, ob und wie digitale Spielwelten solch situationsübergreifende, generische Kompetenzen fördern können. Hier wird man weiter nach Spielkonzepten suchen und die Lerneffekte sehr genau analysieren müssen. Im Folgenden werden zwei Alternativen vorgestellt, mit denen die angestrebten Lernprozesse gezielter eingelöst werden: Die Einbettung des Spiels in eine didaktisierte Lernsituation und die Einbettung von Lernaufgaben in das Spiel.

\subsection{Einbetten in Lernsituationen}

Zur Sicherung eines Lerneffektes kann das Spiel in eine didaktisch aufbereitete Lernsituation eingebettet werden. Statt zu hoffen, dass bereits im Spiel selbst bestimmte Kompetenzen erworben werden, kann das Spiel dadurch didaktisiert werden, dass eine Instruktion für die Spieldurchführung erfolgt, die bestimmte 
Erfahrungen bei den Teilnehmenden induzieren soll, und später ausgewertet wird. Auf diese Weise erfolgt eine didaktische Rahmung des Spiels.

Verschiedentlich wurde bereits darauf hingewiesen, dass eine Auswertung von Erfahrungen im Anschluss an ein Spiel, eine Simulationen o. ä. erforderlich ist, um überhaupt einen Lerneffekt eintreten zu lassen (Crookall 2005; vgl. Garris u.a. 2002). Das Spiel dient der Motivierung, der Emotionalisierung und der Selbsterfahrung; im "De-Briefing» aber, der Auswertung der eigenen Spielerfahrung, so die Überlegung, findet der eigentliche Lernprozess statt. Dewey (1938) etwa kann zitiert werden mit der Forderung "experience plus reflection equals learning»: Die Reflexion einer Erfahrungssituation ist wesentliche Voraussetzung für einen pädagogisch zielgerichteten Lernerfolg.

Bei der Planung entsprechender Lernsituationen ist damit zu bedenken, dass der Spieleinsatz gegenüber einer traditionellen Wissensvermittlung ohne solche involvement-induzierenden Elemente immer mit einem zusätzlichen Zeitaufwand verbunden ist. Zugleich erscheint der Zeitaufwand sehr wohl gerechtfertigt, wenn das Spiel es schafft, eine persönliche Anteilnahme zu erzeugen und einen «Anker» zu setzen für die Reflexion der Lerninhalte.

Ein mögliches Problem kann nun dadurch entstehen, dass die Spielsituation durch die emotionale Ansprache der Teilnehmenden eine Eigendynamik entwickeln kann, die vom eigentlich intendierten Lerninhalt ablenkt. Die Personen sind ganz «im» Spiel gefangen und können sich schwer auf die abstrakte Auswertung einlassen. Insofern könnte gerade eine starke Immersion die didaktische Auswertung erschweren. Hinzu kommt: Das Spiel erfordert immer auch Vorbereitung, Einführung und Einübung und erfordert damit oft mehr Zeit als geplant. Das geht oftmals zulasten der Auswertung; die Gefahr besteht, dass es beim Spielen bleibt und die Reflexion zu kurz kommt.

\subsection{Einbetten von Lernaufgaben}

Ein Spiel kann, wie beschrieben, in eine Lernsituation eingebettet werden. Es können aber auch - umgekehrt - didaktisch aufbereitete Elemente in das Spiel eingebettet werden. Bei dieser didaktischen Konzeption werden Lernaufgaben in den Spielablauf einmontiert. Es sind Aufgaben zu lösen, um weiterspielen zu können, um ein weiteres Spiel-Level zu erreichen oder andere Vergünstigungen oder Punkte zu erzielen. Das Spiel ist quasi die Belohnung für eine richtige Aufgabenlösung.

Die Aufgaben können inhaltlich eng an die Spielhandlung anknüpfen. So ist etwa in einem Physik-Lernspiel der richtige Widerstand einzulegen, damit ein Stromkreis geschlossen werden kann und Licht in eine Schatzkammer gebracht werden kann. Oder es ist zu berechnen, welches Gewicht bei einem Flaschenzug zu wählen ist, damit ein Fass aus einem Schiff entnommen werden kann. Für die Aufgabenlösung kann (und muss in der Regel) auf Informationen aus einem getrennten Wissensteil 
zugegriffen werden. Dieser Wissensteil beinhaltet didaktisch aufbereitete Informationen, die sich die Person aneignen soll bzw. muss, um die Aufgabe zu lösen. In der Regel versuchen Personen solche Aufgaben mit einer Versuch-und-IrrtumStrategie zu lösen. Doch zumeist ist dies nicht zielführend, so dass es zwingend erforderlich wird, sich die relevanten Informationen aus dem Wissensteil zu suchen. Zu bedenken sind die o. g. Anmerkungen zum Erlernen von Spielen. Danach erscheint es möglich, dass ein explizites Umschalten in einen «Lernmodus» von Personen als eher störend erlebt wird, so dass auch hier zu fragen ist, wie man die Informationssuche und -aufnahme möglichst «didaktisch-immersiv» (Bopp) gestalten könnte.
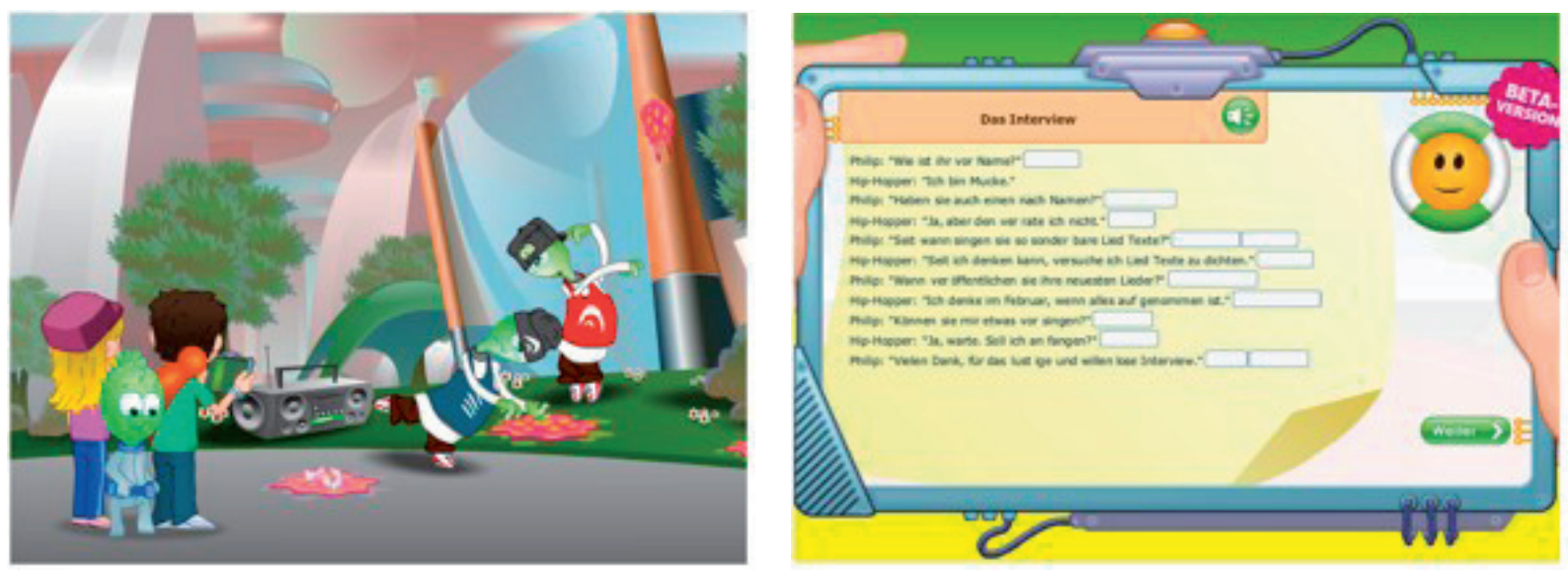

Abb. 2: Problemsituation in die Spielwelt Scoyo (l) mit Lernaufgabe ( $r$ )

Die Aufgaben können auch inhaltlich unabhängig von der Spielhandlung sein. Bei einem Abenteuer im Weltall kann etwa ein Tor nur geöffnet werden, wenn die Person in einem Text Adjektive und Verben richtig identifiziert. Die Aufgabe ist lediglich als Türöffner konzipiert, sie ist nicht inhaltlich mit der Story verbunden. Dies erscheint für die Motivation der Spielenden auf den ersten Blick weniger attraktiv. Die Lösung ist aber insofern interessant, weil während der Laufzeit aus einem Pool von Aufgaben eine passgenaue Aufgabenstellung ausgewählt werden kann in Abhängigkeit von Parametern des Lernstands. Auf diese Weise können Varianten eines adaptiven Lernprogramms im Spiel realisiert werden (s. Leutner 1992).

Für die Internet-Plattform scoyo.de wurde beispielsweise ein solcher Ansatz gewählt. Hier zeigten Beobachtungen von Kindern, dass sie durch diese Aufgaben nicht aus dem Spielfluss gerissen werden, sondern durchaus die Motivation besteht, diese Aufgaben als Bestandteil des Spiels anzuerkennen und zu bearbeiten. 


\section{Zur Differenz von Spiel- vs. Lernmodus}

Die Frage, ob Spiel- und Lernmodus tatsächlich subjektiv als gegensätzliche Zustände erlebt werden, ist für die Konzeption von Lernspielen von grundlegender Bedeutung (s. a. Bopp, 2005). Aus den bisherigen Überlegungen wäre abzuleiten, dass der Wechsel in einen Modus expliziten Lernens in einem Spiel eher vermieden wird, und nur als kurze Unterbrechung akzeptiert wird, um Informationen aufzunehmen, mit denen man sich wieder schnell in den Spielfluss zurückbegibt. Eine intensivere Auseinandersetzung, gar ein beiläufiges Aufnehmen von didaktisch aufbereiteten Wissensbeständen, erscheint nicht zielführend. Der «Lernmodus» behindert das Eintauchen in den Spielfluss und wird subjektiv als Umschalten in ein anderes Skript erlebt. Der Lernmodus bedeutet für die Person, dass sie aktiv in ein anderes Skript umschaltet, und dass sie subjektiv «aus» dem Spiel herausgeht. Sie fühlt sich möglicherweise gezwungen, sich Informationen zu beschaffen, um wieder in das Spiel eintauchen zu dürfen.

Diese Problematik lässt sich durch eine Studie von Bormann et al. $(2008 ; 2007)$ illustrieren, die das Lernspiel «Physikus» zum Gegenstand hatte. Das Lernspiel folgt dem Ansatz der «eingebetteten Lernaufgaben», die inhaltlich eng an die Spielhandlung gekoppelt sind, und für den Spielfortschritt zu lösen sind. So wie bei World-of-Worcraft (WOW) ein bestimmtes Level nur erreicht werden kann, wenn die Person genauere Kenntnisse hat über die Eigenschaften bestimmter Objekte, so kann bei Physikus ein «Abenteuer» nur bewältigt werden, wenn ein bestimmtes Wissen aus der Physik vorliegt.

Untersucht wurde bei Bormann et al. der Umgang mit dem System bei Studierenden. Sie bearbeiteten das Lernspiel (nur) mit dem Hinweis, dass die Studie dazu dient, das Setup zu überprüfen, während ihr Verhalten per Blickbewegungskamera aufgezeichnet wurde. Untersucht wurden drei Inhaltsseiten: (1) die Startseite des Wissensteils, in der allgemeine Informationen über das Thema zu finden ist, (2) eine Wissensseite, mit didaktisch aufbereiteten Informationen zu dem Thema und (3) eine (sehr ähnlich aufgebaute) Formelseite, die erläutert, wie Werte in einer Formel einzusetzen bzw. zu berechnen sind. Für die Aufgabenlösung ist alleine die Formel relevant, in die bestimmte Werte einzusetzen sind. Für das Verständnis der zugrunde liegenden Begriffe, der Abkürzungen und Zusammenhänge ist dagegen die intensivere Rezeption der Wissensseite notwendig. Im Folgenden zeigen wir aus dieser Studie Muster von Blickbewegungen, die illustrieren, dass die Informationen auf der - didaktisch relevanten - Informationsseite eher oberflächlich abgetastet werden. Die Muster können interpretiert werden als Hinweis darauf, dass - über die Formelseite - die notwendigen lösungsrelevanten Informationen erfasst werden, aber sonst weitere Informationen eher "gemieden» werden. Die Wissensseite wird nach Schlagworten abgetastet, grafische Elemente (eyecatcher) werden besonders beachtet, eine tiefere, für das Lernen notwendige Verarbeitung findet 
nicht statt. Insgesamt zeigt sich ein Muster, das dafür spricht, dass die explizite Informationssuche schnell verlassen wird, um erneut in das Spiel einzutauchen.

Fixationen der Formelseite:

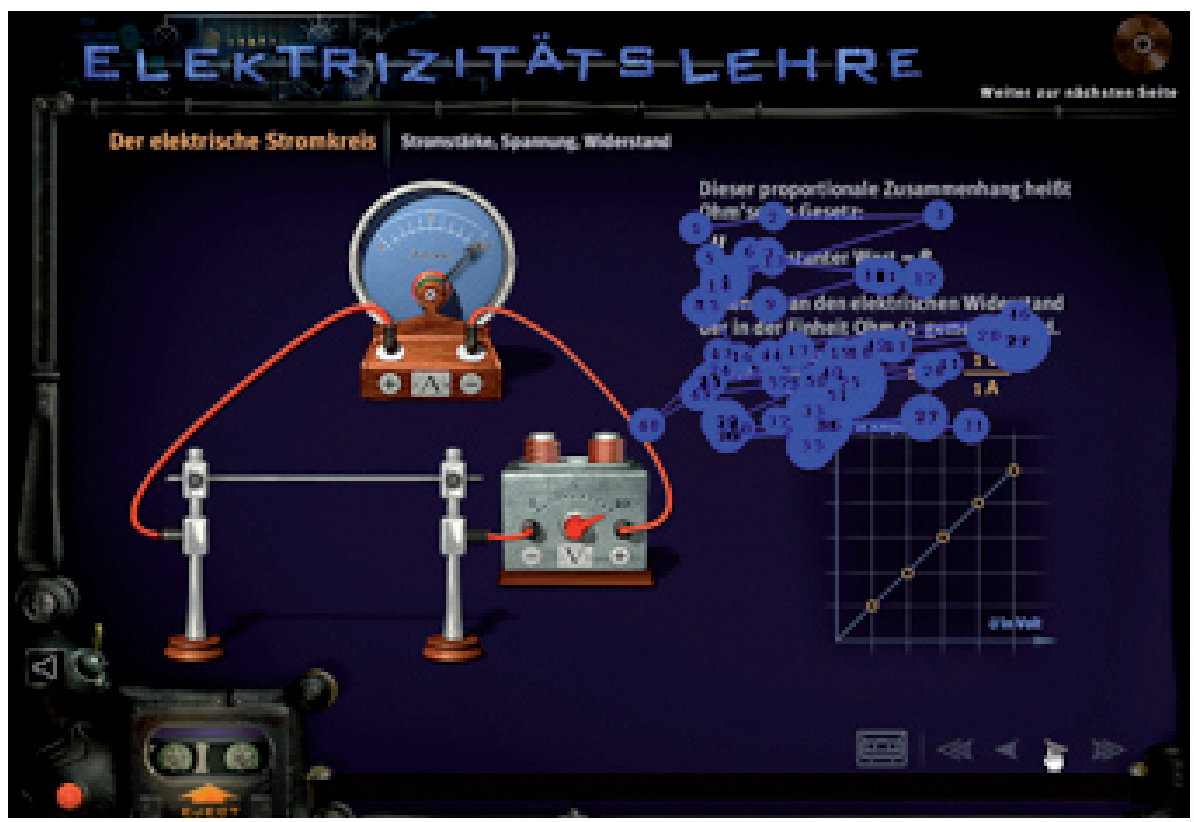

Fixationen der Wissensseite:

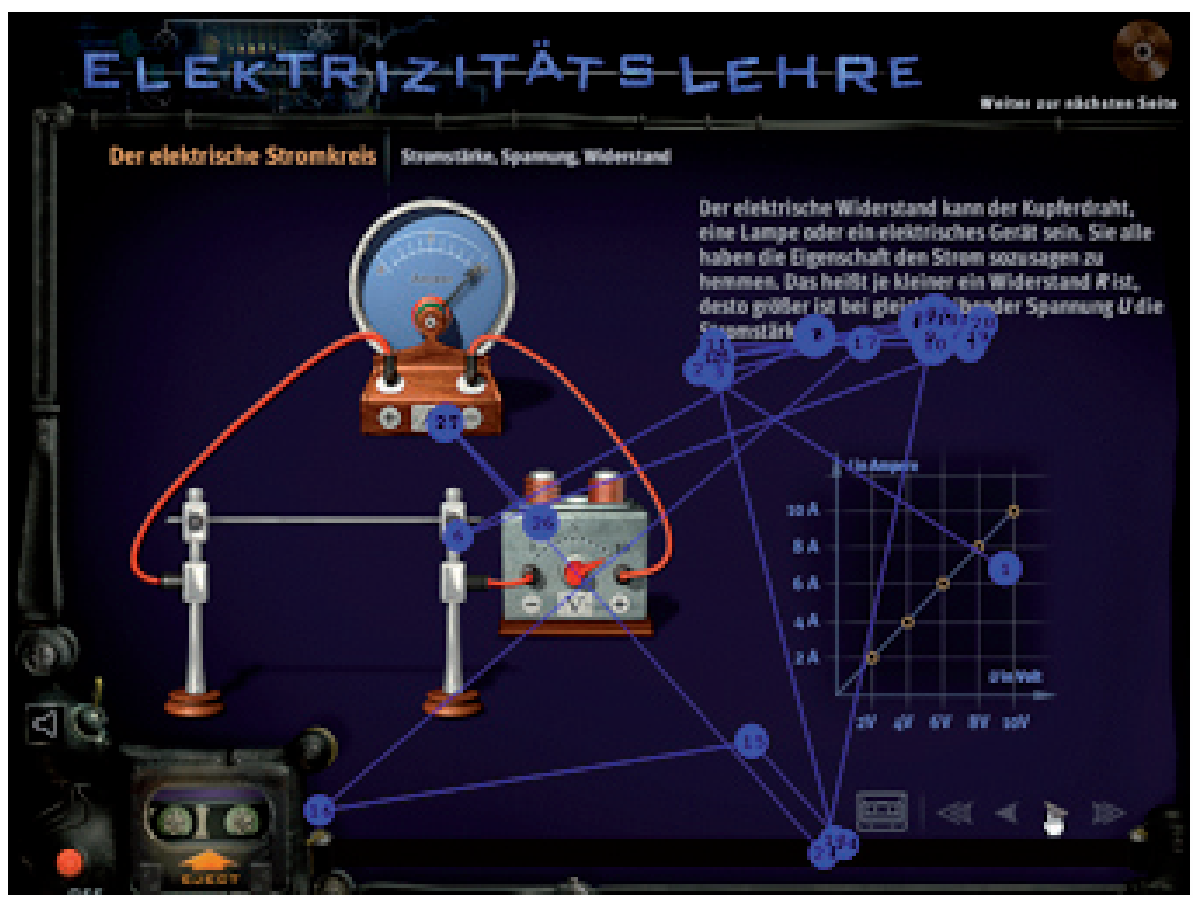


Fixationen der Startseite:

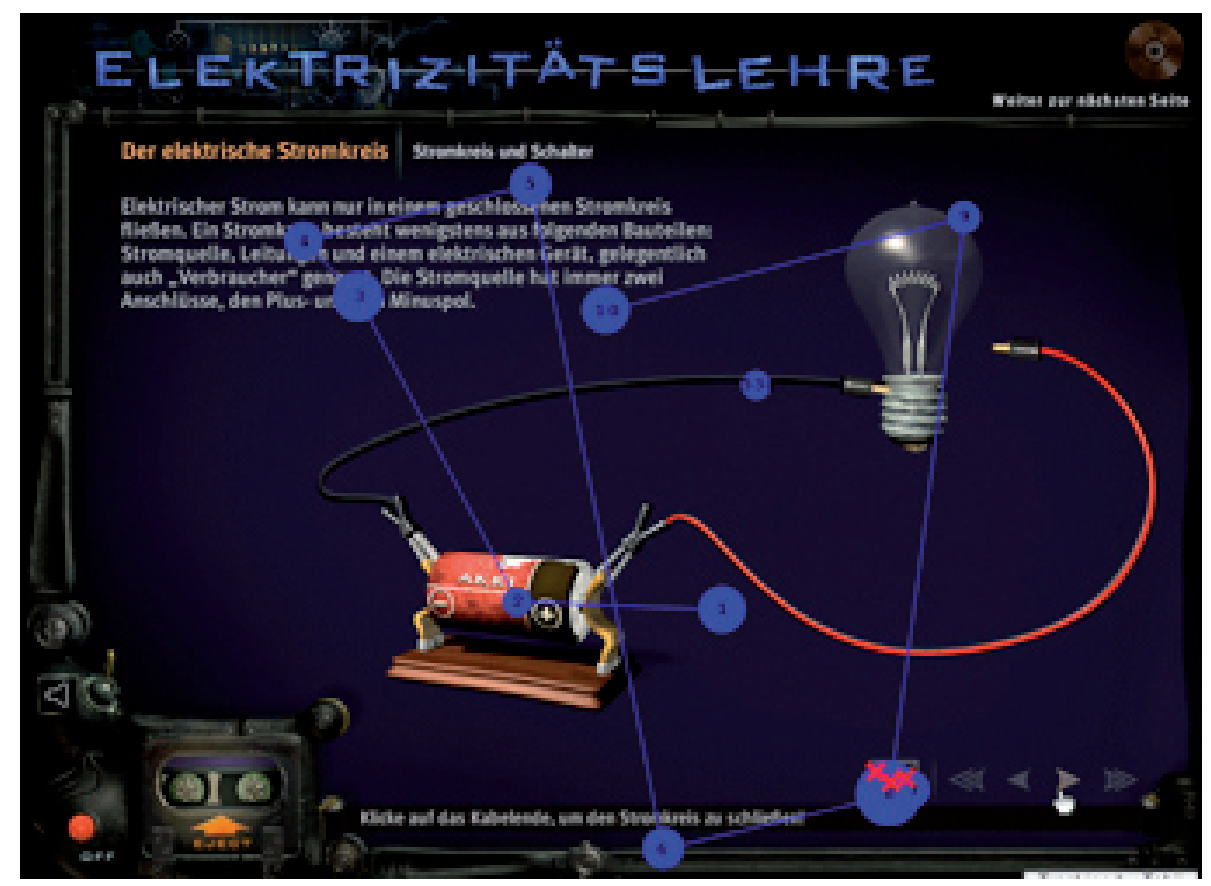

\section{Implikationen für das Didaktische Design}

Zunächst bleibt zu konstatieren, dass den weitreichenden Erwartungen einiger GBL- Protagonisten die Position mancher Kulturtheoretiker des Spiels entgegen steht, wonach Spiele sich einer einfachen Nutzbarmachung für pädagogische Zwecke entziehen: Ein Spiel, das didaktisch instrumentalisiert wird, ist kein Spiel mehr (vgl. etwa Huizinga 2004; Oerter 1999).

Unsere Diskussion hat drei grundsätzliche Varianten der didaktischen Konzeption von digitalen Spielwelten aufgezeigt, die nochmals in der folgenden Übersicht benannt sind. Bei der Konzeption einer GBL-Anwendung ist dabei eine Entscheidung für eine der Varianten zu wählen. Bei Variante (1) ist die Frage der Übereinstimmung von Spiel- und Anwendungssituation zu reflektieren und zu berücksichtigen, ob Wissen über das wirkliche Funktionieren der Anwendungssituation existiert, die bei der Implementation der Spielwelt herangezogen werden kann (wie z. B. bei der Nachbildung technischer Systeme).

Für Spiele, die in eine didaktisierte Lernsituation eingebettet werden, stellen sich dagegen vergleichsweise wenige Anforderungen. Im Prinzip vermag fast jedes Spiel Erfahrungen zu vermitteln, die sich in einer Reflexion auswerten lassen. Hier sind eher die zeitliche Organisation und mögliche sozio-emotionale Nebeneffekte zu beachten. 
Bei der dritten Variante stellt sich die konzeptuelle Herausforderung, wie der Spielfluss durch Einbettung von Lernaufgaben möglichst aufrechterhalten werden kann. Mediale und technische Brüche sind zu vermeiden, damit subjektiv eine Einheit bestehen bleibt.

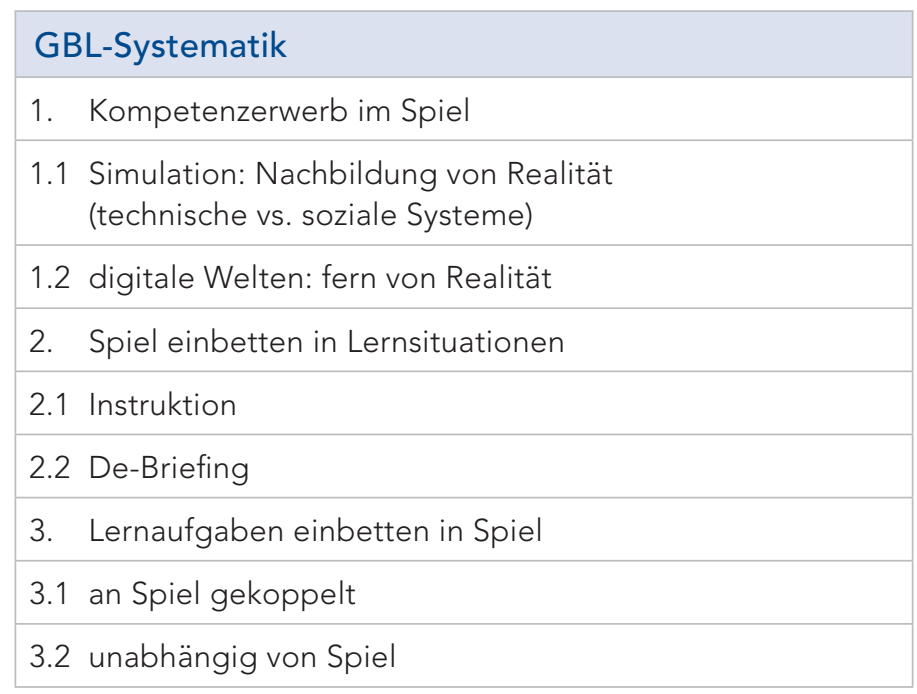

Die Übersicht listet damit verschiedene Varianten, wie digitale Spielwelten für didaktische Zielsetzungen grundsätzlich angelegt werden können. Sie bietet eine Orientierung für die Konzeption von spielerischen Lernangeboten. Die Analyse der Varianten hat gezeigt, das die didaktische Nutzbarmachung von Spielwelten eine Herausforderung für das Instruktionsdesign wie das Spieldesign gleichermassen darstellt (s.a. Wagner 2008 zum Verhältnis von Instruktions- und Game Design)' ${ }^{1}$. Bislang existieren eher wenige Beispiele, in denen eine Integration von Spielen und Lernen überzeugend umgesetzt ist. Es erscheint notwendig, weiter über die Nutzbarmachung von Spielen für das Lernen nachzudenken und innovative Lösungswege zu erproben. Dabei wird auch die Reichweite deutlicher werden, die mit den verschiedenen Varianten von Spielwelten verbunden ist, welche Lerninhalte und -ziele damit verfolgt werden können und was kritische Erfolgsfaktoren sind, die bei den einzelnen Varianten zu beachten sind.

Die empirische Befundlage zu Lerneffekten von Spielen kann hier nicht näher diskutiert werden. Viele Studien helfen u. E. eher begrenzt weiter, weil sie auf Vergleichen basieren, die eine Versuchsgruppe «mit Spiel» einer Gruppe «ohne Spiel» gegenüberstellen. Es überrascht nicht, dass hierbei die «Überlegenheit von GBL bislang nicht nachgewiesen ist», wie die Metaanalysen u. a. von Squirre (2003), Kirriemuir \& McFarlane (2004), Vogel et al. (Jennifer J. Vogel u. a. 2006) oder Arnseth (2006) immer wieder feststellen. Petko (2008) fordert: «Nötig wäre statt dessen

Teilweise wird auch die Frage gestellt, inwiefern Instruktionsdesign und Game Design überhaupt vereinbar ist (vgl. Crosby 2005; Dickey 2006; Fromme 2007; Schrammel und Mitgutsch 2009). 
eine verstärkte Orientierung am «Ausprobieren» der postulierten Potenziale in Bildungskontexten» (S. 11).

\section{Literatur}

Arnseht, Hans Christian (2006). Learning to play of playing to learn. A critical account of the models of cimmuniation informing educational research in computer gameplay. The international Journal of Computer Game Research, 6(1).

Behr, Katharina-Maria; Klimmt, Christoph; Vorderer, Peter (2008). Leistungshandeln und Unterhaltungserleben im Computerspiel. In Thorsten Quandt, Jeffrey Wimmer u. Jens Wolling (Hrsg.), Die Computerspieler. Studien zur Nutzung von Computergames. Wiesbaden: VS Verlag für Sozialwissenschaften. S. 225-240. http://dx.doi.org/10.1007/978-3531-90823-6_13 (Zugriff: 28.2.2009).

Blötz, Ulrich. (2002). Planspiele in der beruflichen Bildung: Abriss zur Auswahl, Konzeptionierung und Anwendung von Planspielen. Bielefeld: WBV.

Bopp, Mathias (2008). Storytelling und parasoziales Design als Motivationshilfen in Computerlernspielen. MedienPädagogik 15/16: http://www.medienpaed.com/15/bopp0812. pdf (10.12.08).

Bopp, Mathias (2005). Immersive Didaktik: Verdeckte Lernhilfen und Framingprozesse in Computerspielen. kommunikation@gesellschaft, 6, 2-12.

Bormann, Mark; Heyligers, Karin; Kerres, Michael; Niesenhaus, Jörg (2008). Spielend Lernen!? Eine empirische Annäherung an die Möglichkeit einer Synthese von Spielen und Lernen.» In S. Seehausen, S. Lucke, \& Firscher (Hrsg.), Lecture Notes in Informatics. LNI Proceedings der 6. elearning Fachtagung Informatik (DeLFI 2008), Sep. 2008. Bonn: Gesellschaft für Informatik.

Bormann, Mark; Kerres, Michael; Vervenne, Marcel (2007). Informationsrezeption und Informationsvermeidung beim Game-Based Learning: eine Studie zum Lernadventure «Physikus». Gehalten auf der Fachgruppe Medienpsychologie der DGPs, TU Dresden.

Crookall, David (2005). A guide to the literature on simulation/gaming. In David Crookall u. Kiyoshi Arai (Hrsg.), Simulation and gaming across disciplines and cultures: ISAGA at a watershed. Thousand Oaks, CA: Sage. S. 151-177.

Crosby, William (2005). Instructional Design does not equal Game Design - Lessons learned in delivering a course in game design and education. In Piet Kommers u. Griff Richards (Hrsg.), Proceedings of World Conference on Educational Multimedia, Hypermedia and Telecommunications. Chesapeake, VA: AACE. S. 2617-2621.

Dickey, Michele D. (2006). "Ninja Looting» for instructional design: the design challenges of creating a game-based learning environment. In ACM SIGGRAPH 2006 Educators program. Boston, Massachusetts: ACM. S. 17. http://portal.acm.org/citation. cfm?id=1179313 (Zugriff 20.2.2009).

Fromme, Johannes (2007). Spiele in virtuellen Umgebungen. Überlegungen zur Beschreibung und Analyse eines neuen Mediums. In D. Hartwich, C. Swertz, M. Witsch, \& Meder (Hrsg.), Mit Spieler : Überlegungen zu nachmodernen Sprachspielen in der Pädagogik. Würzburg: Könighausen \& Neumann.

Garris, Rosemary; Ahlers, Robert; Driskell, James E. (2002). Games, Motivation, and Learning: A Research and Practice Model. Simulation Gaming, 33(4), S. 441-467.

Gee, James Paul (2003). What video games have to teach us about learning and literacy. New York: Plagrave Macmillan. 
Geilhardt, Thomas; Mühlbradt, Thomas (1995). Planspiele im Personal- und Organisationsmanagement. Göttingen: Verlag für Angewandte Psychologie.

Hagel, John; Brown, John Seeley (2009, Januar 14). How World of Warcraft Promotes Innovation. BusinessWeek: Innovation. http://www.businessweek.com/innovate/content/ jan2009/id20090114_362962_page_2.htm (27.2.2009).

Heckhausen, Heinz (1989). Motivation und Handeln. Lehrbuch der Motivationspsychologie. Berlin: Springer.

Huizinga, Johan (2004). Homo Ludens. Vom Ursprung der Kultur im Spiel. Reinbek: Rowohlt.

Kirriemuir, John; McFarlane, Anagela (2004). Literature Review in Games and Learning. Bristol.

Klimmt, Christoph (2005). Computerspielen als Handlung. Dimensionen und Determinanten des Erlebens interaktiver Unterhaltungsangebote. Köln: Halem.

Leutner, Detlef (1992). Adaptive Lehrsysteme. Instruktionspsychologische Grundlagen und experimentelle Analysen. Weinheim: Beltz.

Mähler, Claudia; Stern, Elsbeth (2006). Transfer. In Dieter Rost (Hrsg.), Handwörterbuch Pädagogische Psychologie (S. 782-793).

Mandl, Heinz; Keller, Christel; Reiserer, Markus; Geier, Boris (Hrsg.). (2001). Planspiele im Internet. Konzepte und Praxisbeispiele für den Einsatz in Aus- und Weiterbildung (Bd. 26). Bielefeld: WBV.

Mandl, Heinz; Kopp, Brigitta (2006). Blended Learning: Forschungsfragen und Perspektiven. München, LMU Forschungsbericht, 182.

Oerter, Rolf (1999). Psychologie des Spiels: Ein handlungstheoretischer Ansatz (Durchges. Neuausgabe.). Beltz.

Petko, Dominik (2008). Unterrichten mit Computerspielen. Didaktische Potenziale und Ansätze für den gezielten Einsatz in Schule und Ausbildung. MedienPädagogik, (Themenheft 15/16: Computerspiele und Videogames in formellen und informellen Bildungskontexten). Abgerufen von http://www.medienpaed.com/15/petko0811.pdf.

Prensky, Marc (2003). Digital game-based learning. Comput. Entertain 1(1): S. 21-21.

Salen, Katie; Zimmerman, Eric (2003). Rules of Play: Game Design Fundamentals. MIT Press.

Schank, Robert C.; Abelson, Robert P. (1977). Scripts, plans, goals, and understanding. Hillsdale, NJ: Lawrence Erlbaum Associates.

Schrammel, Sabrina; Mitgutsch, Konstantin (2009). Computerspielen als medial-kulturelle Praktik. Ein medienpädagogisch-kulturtheoretischer Zugang zum Phänomen Computerspielen. MedienPädagogik 15/16: http://www.medienpaed.com/15/schrammel_mitgutsch0904.pdf (30.3.09).

Squire, Kurt D. (2003). Video games in education. International journal of intelligent simulations and gaming, 2(1).

Vogel, Jennifer J. et al. (2006). Computer Gaming and Interactive Simulations for Learning: A Meta Analysis. Journal for Educational Computing Research, 34(3): S. 229-243.

Wagner, Michael G. (2008). Serious Games: Spielerische Lernumgebungen und deren Design. In Ludwig J. Issing u. Paul Klimsa (Hrsg.), Online-Lernen - Handbuch für das Lernen mit Internet. München: Oldenbourg.

Wolling, Jens; Quandt, Thorsten; Wimmer, Jeffrey (2008). Warum Computerspieler mit dem Computer spielen. In Thorsten Quandt, Jeffrey Wimmer u. Jens Wolling (Hrsg.), Die Computerspieler. Studien zur Nutzung von Computergames. VS Verlag für Sozialwissenschaften. S. 13-21. http://dx.doi.org/10.1007/978-3-531-90823-6_1 (Zugriff 17.3.09). 